\title{
Criatividade humana no Oriente e no Ocidente: Análise das raizes históricas-conceituais
}

\author{
Marina Porto-Ribeiro \\ Denise de Souza Fleith
}

\section{RESUMO}

A criatividade é discutida e pesquisada em várias partes do mundo, embora não haja uma definição consensual do termo em âmbito acadêmico. Dado que o fenômeno criativo é interativo e dependente do contexto, variadas culturas o definem, estimulam e reconhecem diferentemente. Uma definição compartilhada por diversos autores americanos descreve a inovação e a adequação como características inerentes à criatividade; ao passo que, entre autores chineses, destaca-se a moral como atributo imprescindível. 0 objetivo deste artigo foi analisar as raízes históricas do estudo da criatividade, derivadas dos conceitos predecessores de criatividade divina no Ocidente e de criatividade natural no Oriente, apontando possíveis influências para concepções e pesquisas atuais. Embora a importância da cultura tenha sido reconhecida nos estudos sobre criatividade a partir dos anos 70, ela ainda é subestimada, especialmente quando se valem da definição psicométrica e de instrumentos americanos para medir a criatividade de cidadãos de variadas nacionalidades. As perspectivas historiométrica e de sistemas são apontadas como opções teórico-metodológicas que reconhecem a criatividade como fenômeno social e podem ser mais adequadas para investigações interculturais.

Palavras-chave: criatividade; cultura; criatividade intercultural; oriente; ocidente

\section{ABSTRACT}

\section{Human creativity in the East and in the West: Analysis of historical-conceptual roots}

Creativity is discussed and studied around the world, even though there is no consensual definition of the term in the academic area. Given that the creative phenomenon is interactive and context-dependent, different cultures define, stimulate and recognize it differently. A definition shared by several American authors describes innovation and appropriateness as characteristics inherent to creativity; whereas, among Chinese authors, morality stands out as an essential attribute. The purpose of this article was to analyze the historical roots of the study of creativity, derived from the predecessor concepts of divine creativity in the West and from natural creativity in the East, pointing possible influences to current conceptions and researches. Although the importance of culture has been recognized in studies of creativity since the 1970s, it is still underestimated, especially when the psychometric definition and North American instruments are used to measure the creativity of citizens of various nationalities. The historiometric and systems perspectives are pointed as theoreticalmethodological options that recognize creativity as a social phenomenon and may be more suitable for intercultural investigations.

Keywords: creativity; culture; intercultural creativity; West; East.

A habilidade de inovar e ser criativo é citada como uma das mais importantes competências do século XXI (Bellanca \& Brandt, 2010; Csikszentmihalyi \& Wolfe, 2000; Trilling \& Fadel, 2009). Nutrir a criatividade parece ser o caminho para enfrentar os desafios, cada vez mais complexos, que a sociedade apresenta. Pesquisas revelam que o desenvolvimento criativo na

\section{Sobre as Autoras}

M.P.R.

orcid.org/0000-0002-8634-9240

Universidade de Brasília (UnB) -

Brasília, DF

marinaportopsi@gmail.com

D.S.F.

orcid.org/0000-0001-7512-8023

Universidade de Brasília (UnB) -

Brasília, DF

fleith@unb.br

\section{Direitos Autorais}

Este é um artigo de acesso aberto e pode ser reproduzido livremente, distribuído,

transmitido ou modificado, por qualquer pessoa desde que usado sem fins comerciais. 0 trabalho é disponibilizado sob a licença Creative Commons CCBY-NC.

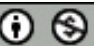




\section{-4* INTERACÃO EM F PSICOLOGIA}

infância é um importante indicador para a realização pessoal e o sucesso e, em última instância, para o desenvolvimento da sociedade (Runco, Millar, Acar, \& Cramond, 2010; Sternberg, Jarvin, \& Grigorenko, 2009).

Definir a criatividade, no entanto, não é tarefa simples. De acordo com Alencar e Fleith (2009), apesar dos esforços envidados por pesquisadores desde 1950, quando o então presidente da Associação Americana de Psicologia J. P. Guilford os convocou para a reflexão científica sobre o conceito, não há consenso acerca do exato significado desse termo. Inicialmente, em uma perspectiva mais direcionada ao indivíduo criativo, foram publicados múltiplos estudos com o objetivo de verificar habilidades de pensamento e traços de personalidade associados à criatividade (Barron, 1955; Guilford, 1967; MacKinnon, 1962). Há ainda outra perspectiva de investigação direcionada à criatividade de animais não humanos (Kaufman, Butt, Kaufman, \& Colbert-White, 2011). Neste artigo, no entanto, é discutida apenas a criatividade humana.

De acordo com Glaveanu (2010), na maioria dos estudos realizados até a década de 70 , o indivíduo era analisado separadamente do contexto social e cultural. A partir dos anos 70, no entanto, a desatenção ao contexto nos estudos sobre criatividade foi reconhecida como um viés do campo (Hennessey, 2003). Vários sistemas sociais e culturais influenciam a criatividade: a família, a escola, o trabalho, a comunidade local, o país (Klausen, 2010; Lubart, 2010). A criatividade, portanto, é um fenômeno contextual. Um tópico amplamente discutido na psicologia é a influência da cultura para a criatividade (Lau, Hui, \& Ng, 2004; Lubart, 1999; Niu \& Sternberg, 2002; Rudowicz \& Yue, 2000). Todavia, de acordo com Lubart (2010), apesar de sua importância, a cultura é muitas vezes desconsiderada em toda sua amplitude no estudo da criatividade:

A cultura é onipresente, e por essa razão seu impacto é normalmente subestimado. A cultura fornece a base, a estrutura psicológica profunda em que toda a atividade humana ocorre. Para atividades complexas com facetas sociais, como a criatividade, a importância de compreender a influência da cultura é particularmente importante. (p. 276)

Em culturas de diferentes nações pode-se identificar percepções distintas, ou até mesmo conflituosas, sobre o que é criatividade. Em consequência dos variados conceitos, um fenômeno ou indivíduo criativo pode ser estimulado e reconhecido em determinada cultura mas não em outra. Além disso, mesmo considerando uma definição específica de criatividade, essa habilidade poderia ser desenvolvida diferentemente em indivíduos sob influência de culturas de uma ou várias nações (Porto-Ribeiro \& Fleith, no prelo; Wong \& Niu, 2013).
Marina Porto-Ribeiro e Denise de Souza Fleith
Tsang e Prendergast (2009) destacam o interesse de pesquisadores pelas abordagens orientais e ocidentais do conceito. Embora a divisão ocidente-oriente seja abrangente, já que cada um dos polos abarca uma variedade de culturas (Lan \& Kaufman, 2012), uma análise da evolução conceitual da criatividade nesses dois polos fornece insumos que podem ser relacionados às discussões atuais. Ademais, a análise das influências culturais enceta a reflexão sobre o quanto as pesquisas em criatividade (como, por exemplo, as que buscam comparar o desempenho criativo de indivíduos de diferentes nacionalidades) podem estar enviesadas pela perspectiva norte-americana (Kaufman \& Sternberg, 2006).

\section{BREVE HISTÓRICO DO DEBATE SOBRE CRIATIVIDADE NO OCI- DENTE E NO ORIENTE}

Niu e Sternberg (2006) analisaram as raízes filosóficas dos conceitos de criatividade nas culturas ocidental e oriental, destacando o quanto as concepções se modificaram ao longo dos anos. No Ocidente houve pelo menos dois tipos de entendimento sobre criatividade: a divina e a individual. A princípio, a criação e a inovação eram atribuídas exclusivamente à inspiração divina e, mesmo quando materializadas pelo homem, ainda eram consideradas dádivas de um Deus ou de muitos deuses. Essas concepções estariam relacionadas, inicialmente, à mitologia grega, segundo a qual as musas seriam responsáveis pelas invenções e artes e, posteriormente, à explicação bíblica de que Deus criou o céu e a terra. A criatividade, em ambos os casos, viria de uma entidade fora do homem (Niu \& Sternberg, 2006).

Nessa concepção divina de criatividade, os homens não teriam potencial para criar algo novo. De acordo com Krausz (2007), a poesia e a música na Grécia antiga eram expressões sobre-humanas. As musas eram as patronas de toda beleza e santidade, que elegiam homens comuns para se tornarem poetas, conhecedores do sublime. De forma análoga, na descrição bíblica de Gênesis, não somente o homem é criação divina, como também o próprio mundo. Deus criou tudo, a partir do vazio ou do nada. Sua criação representaria toda a bondade, inclusive a moral (Weiner, 2000). Com base nessas duas tradições ocidentais, a criatividade emanaria sempre de Deus e dos deuses. Segundo Niu e Sternberg (2006), o conceito de criatividade divina prevaleceu no Ocidente por muito tempo, para além do período artístico do Renascimento. Muitos produtos considerados criativos desse período, na pintura, na escultura e na arquitetura, estavam relacionados à igreja ou à Bíblia, como, por exemplo, os afrescos do teto da Capela Sistina, no Vaticano, concebidos por Michelangelo.

Já o lluminismo foi um período de grande influência sobre a mudança de perspectiva da criatividade divina para a criatividade individual. Nessa fase, de acordo com Albert e Runco 


\section{WIN INTERACÃO EM ET PSICOLOGIA}

(1999), as pessoas começaram a exaltar direitos e poderes individuais para entender o universo e dirigir seu próprio destino. Segundo os autores, novas descobertas surgiram em diversas áreas, especialmente em ciência e tecnologia, e o produto criativo começou a ser aceito como um feito do indivíduo, originado do pensamento e da habilidade humana de criar. Progressivamente, a pessoa criativa se tornou valorizada e, conforme destacam Sternberg e Lubart (1995), diante das perspectivas individualistas da sociedade, ser criativo tornou-se uma forma de se destacar na multidão.

Glaveanu (2010) discute a evolução do conceito de criatividade a partir de paradigmas que podem ser relacionados às raízes filosóficas do Ocidente, sinalizando inclusive um cenário posterior à criatividade individual. 0 autor argumenta que, antes do século 20, discutia-se o conceito do gênio solitário (o Paradigma do Ele), exclusivo, desconectado e inatingível. Esse paradigma refere-se estritamente a criações de alto nível e teria emergido na Grécia e na Roma antiga. 0 incomum gênio solitário talvez estivesse mais próximo dos deuses do que de um ser humano. Segundo o autor, a partir de 1950, a criatividade individual passa a ser discutida na perspectiva de um solitário normal (o Paradigma do Eu), como uma realização humana cognoscível. No segundo paradigma, é abordado um amplo espectro de níveis de criatividade, desde feitos cotidianos até grandes invenções. O paradigma do Eu, no entanto, incentivou uma linha de investigação sobre criatividade focada na cognição e na personalidade individual diante de um vácuo social.

Para Glaveanu (2010), há ainda um terceiro paradigma que reflete o entendimento dos pesquisadores contemporâneos. 0 autor descreve o potencial criativo explorado na relação com o outro (o Paradigma do Nós), em meio a estruturas sociais e individuais. A partir do paradigma do Nós, Glaveanu argumenta que a criatividade deve ser entendida como fenômeno grupal, que se desenvolve dentro das barreiras dos ambientes cultural, comunitário, político e social, mensurado no contexto de seus vastos sistemas complexos. Em concordância com a evolução do entendimento sobre criatividade, Alencar e Fleith (2009) afirmam que a partir da década de 80, esse constructo passa a ser considerado um fenômeno sociocultural. Teorias que exemplificam esse cenário são: o Modelo Componencial de Criatividade (Amabile, 1996), a perspectiva historiométrica (Simonton, 1994), a de sistemas (Csikszentmihalyi, 1988), entre outras.

No Oriente, ou mais especificamente na China, as antigas concepções de criatividade remetem a algo natural, não divino, vez que grande parte dos chineses não adoram um deus personalizado, análogo ao conceito ocidental (Niu \& Sternberg, 2006). Cheng (1991) argumenta que os chineses já acreditaram em uma autoridade moral supernatural e no potencial criador e julgador, por volta de 1200 AC. Essa autoridade era chamada de Tian (Céu). No entanto, segundo o autor, a ideia da personificação divina teria desaparecido com o Taoísmo clássico, antes de $480 \mathrm{AC}$, e não teria tido tanto impacto no pensamento chinês quanto o yin-yang ou o dao, termos que designam a suprema força da natureza e representam a origem de tudo. Esses elementos do taoísmo tornaram-se ortodoxos na China, eventualmente suplantando a crença personificada anterior. Assim, uma das concepções mais difundias entre os chineses é de que as infınitas mudanças e interações do yin-yang deram origem ao dao e teriam originado o mundo, a natureza e toda a bondade, incluindo a moral. Como parte do todo, o potencial criativo também seria originado pelas forças naturais.

Os orientais raramente abordam diretamente a criatividade individual, talvez como uma forma de preservar as tradições coletivistas, ou porque o conceito não se diferencie tanto da criatividade natural (Niu \& Sternberg, 2006). Ao longo da história da filosofia chinesa, um entendimento compartiIhado pela maioria das escolas é a unidade da natureza com o ser humano (Cheng, 1991), de tal forma que todos os princípios da natureza poderiam ser aplicados ao homem. Assim, a filosofia chinesa apresenta uma rica discussão sobre como a criatividade pode ser atingida por meio da experiência e da interação com a criatividade natural. O Taoísmo, por exemplo, sugere que, para se atingir a criatividade, a pessoa deve se aproximar do reino da quietude e do reino do não ser humano, acessando suas próprias raízes naturais e espirituais, preservando o dao (Chang, 1970).

Para se alcançar um alto nível de criatividade, segundo Chang (1970), as pessoas devem se libertar de qualquer conhecimento antigo e entrar em um estado no qual tudo perde sua individualidade e se funde, um estágio de perder a si mesmo, chamado nível invisível de simpatia. Atingindo esse ponto, o indivíduo está livre para se conectar ao universo e ser altamente criativo. Para essa concepção de criatividade, quanto mais as fronteiras da individualidade estiverem apagadas, quanto menos o indivíduo estiver consciente de sua unidade, mais rico será o resultado do processo criativo. $\mathrm{Na}$ história chinesa, grandes poetas e artistas, por meio da meditação e do autocultivo, atingiram esse estado e produziram obras importantes e famosas (Chang, 1970).

Niu e Sternberg (2006) comparam o recurso ancestral chinês de atingir o nível invisível de simpatia com a teoria moderna do flow no processo criativo, proposta por Csikzentmihalyi $(1988,1996)$. O flow é descrito como um estado subjetivo de atenção concentrada intensa, no qual, paradoxalmente, há perda da autoconsciência e distorção da 


\section{MI* INTERACÃO EM LF PSICOLOGIA}

percepção temporal. Durante o flow, o indivíduo opera em capacidade máxima e a experiência da atividade funciona como recompensa intrínseca, sendo o objetivo final meramente um pretexto para o processo (Nakamura \& Csikzentmihalyi, 2014). Quando as pessoas se engajam a ponto de atingir um estado de flow, não conseguem perceber o que está acontecendo fora delas. Adicionalmente, o fato do flow poder ser vivenciado por meio de diferentes tipos de meditações orientais (Nakamura \& Csikzentmihalyi, 2014) corrobora a comparação levada a cabo por Niu e Sternberg (2006).

As concepções de criatividade iniciam-se com as ideias de criatividade divina e natural, no Ocidente e no Oriente. Temse similaridades e diferenças nos conceitos históricos. Niu e Sternberg (2006) destacam duas características que diferenciam as criatividades divina e natural. A criatividade divina ocidental, sempre esteve vinculada à inovação, fator que não necessariamente está presente no entendimento da criatividade natural chinesa. De outro lado, a criatividade natural remete a algo eminentemente bom, ao passo que o conceito de criatividade divina também pode produzir coisas ruins ou imorais.

Tais concepções filosóficas e históricas de criatividade divina e criatividade natural tiveram impacto nas concepções atuais em cada cultura e entre culturas, influenciando uma e outra. Por meio de definições implícitas mais modernas é possível verificar que as diferenças históricas ainda permeiam o conceito de criatividade. Definições implícitas são crenças do senso comum sobre um tema. Contrariamente às definições explícitas, são conceituais ao invés de empíricas (Lan \& Kaufman, 2012). Em um clássico estudo desenvolvido por Sternberg (1985), foi solicitado que americanos, leigos e professores, indicassem comportamentos característicos de uma pessoa criativa, inteligente e sábia, com o objetivo de descobrir as definições implícitas dessas características. Foram mencionadas características de pessoas imaginativas, que questionam normas sociais, têm bom gosto estético e bom humor.

Por outro lado, Rudowicz e Hui (1997) verificaram que traços como humor e bom gosto estético foram negligenciadas nas concepções de criatividade em Hong Kong. Nesse estudo, a pessoa criativa era descrita como alguém que produz uma contribuição para a sociedade, aspecto ausente na amostra americana. Rudowicz e Yue (2000) também evidenciaram que bom gosto estético e humor eram raramente mencionados na concepção de estudantes universitários da república da China, de Hong Kong e de Taiwan sobre criatividade.

Lan e Kaufman (2012) apresentaram um exemplo dessa diferença de percepção sobre a criatividade. Um desenho sarcástico sobre política ou sobre um líder do governo pode ser visto como divertido e criativo na América, mas pode ser desrespeitoso e inconveniente no cenário chinês. Estudos americanos contemporâneos têm discutido também o lado negativo da criatividade, que produz ideias ou comportamentos imorais, antiéticos ou maléficos (Runco, 2017). Gino e Ariely (2012) evidenciaram, por exemplo, a relação entre criatividade e desonestidade. Eisenman (2010) analisou casos de criminosos que usaram a criatividade para obter êxito, ao passo que Cropley (2010) chamou a atenção para o uso da criatividade em atos terroristas.

A partir da revisão de outros estudos, Hondzel e Gulliksen (2015) afirmam que países ocidentais, de estrutura social individualista, tendem a enfatizar o produto criativo e usam a inovação e a adequação de uso como critérios para julgar a criatividade. Já a cultura oriental, que tende ao coletivismo, valoriza a criatividade como aspecto de realização pessoal, com orientação e reverência de autoridades e tradições do passado.

$\mathrm{Li}$, em um trabalho publicado em mandarim (citado por Lan \& Kaufman, 2012), argumenta que os chineses vinculam criatividade a padrões éticos e morais. Os produtos e serviços criativos devem ter valor simbólico, significado social e uma conotação cultural. Os chineses tendem a conceituar a criatividade como uma atitude mais socialmente relacionada, que obedece a normas culturais e proporciona benefícios para a sociedade. Trata-se de uma concepção mais dinâmica sobre a criatividade, que pode envolver o reuso e a reinterpretação ao invés da quebra da tradição (Lubart, 2010).

Diferentemente das definições implícitas, as definições explícitas de criatividade no Ocidente e no Oriente apresentam maior similaridade (Lan \& Kaufman, 2012). Definições explícitas são descritas por psicólogos ou especialistas e geralmente são baseadas em dados científicos. Importantes teóricos americanos concordam que as características de inovação/originalidade e utilidade/adaptabilidade são críticas na definição de criatividade (Amabile, 1983; Barron, 1955; Sternberg \& Lubart, 1999; Torrance, 1988). De acordo com Li (citado em Lan \& Kaufman, 2012), dentro da comunidade de pesquisa chinesa, as definições iniciais de criatividade eram simplesmente a aplicação de teorias americanas no contexto cultural chinês.

Lan e Kaufman (2012) destacam que, com a expansão da psicologia social e o aumento das pesquisas transculturais, conceituações mais culturalmente específicas surgiram na China. Um estudo publicado em chinês por Mao, Guo, Chen e Lin, (citado em Lau \& Kaufman, 2012, p. 288), apresenta oito características da criatividade: "criar um produto que nunca foi criado antes, viver uma vida criativa, resolver um problema, combinar fatores e construir novas relações, ter a capacidade de ser criativo, ter personalidade criativa, pensar com 


\section{-3: INTERACÃO EM LF PSICOLOGIA}

criatividade e ter um desempenho abrangente". A definição chinesa pode parecer complexa, talvez porque parte de uma lógica diferente da que se aplica à cultura ocidental.

Portanto, ao longo dos anos, diversos pesquisadores buscam definir o conceito de criatividade, nos Estados Unidos, na China, no Ocidente, no Oriente. Como argumentam Lan e Kaufman (2012), mesmo que haja concordância sobre algumas características que definem a criatividade, o significado dessas características pode variar de acordo com as culturas. Até a tradução do termo é um aspecto a ser considerado. Por exemplo, há duas expressões chinesas para se referir ao conceito de criatividade, mas nenhuma é aplicável à discussão sobre produto criativo.

As variadas definições de criatividade podem tornar-se um viés de pesquisa no cenário acadêmico, por exemplo, em comparações entre o desempenho criativo de indivíduos de diferentes nacionalidades (Kaufman \& Sternberg, 2006). Medir a criatividade descrita pelo paradigma americano psicométrico em um cenário oriental pode ser injusto vez que, neste último, o potencial criativo é entendido e desenvolvido diferentemente. Outras metodologias podem ser mais adequadas, como, por exemplo, a historiométrica, na qual a criatividade é mensurada submetendo dados históricos e biográficos a análises objetivas e quantitativas (Simonton, 1994). Vez que essa perspectiva é centrada na trajetória de realizações e reputação individual, e parte do conceito de criatividade como fenômeno social, é aplicável em diferentes nações e culturas.

\section{O DESEMPENHO CRIATIVO EM DIFERENTES PARTES DO MUNDO}

Zha, Walczyk, Griffith-Ross, Tobacyk e Walczyk (2006) buscaram analisar o desempenho criativo de adultos americanos e chineses, considerando as influências de suas culturas, descritas como individualistas e coletivistas, respectivamente. Os resultados indicaram índice criativo significativamente maior dos americanos em relação aos chineses, e que o primeiro grupo detinha alto grau de individualismo e o segundo, de coletivismo. Os autores sugeriram que o individualismo facilitaria o desempenho criativo nas culturas ocidentais e que o coletivismo dificultaria a expressão criativa no Oriente. Kaufman e Sternberg (2006) ponderam, no entanto, que o estudo de Zha et al. (2006) valeu-se do instrumento Teste Torrance de Pensamento Criativo, que mede o conceito de criatividade psicométrica definido pelos americanos, e sugerem uma reflexão sobre quão enviesadas pelo paradigma ocidental estariam as pesquisas em criatividade.

Outros estudos comparativos mais recentes, como o de Kim, Lee, Chae, Anderson e Laurence (2011), também adota-
Marina Porto-Ribeiro e Denise de Souza Fleith

ram apenas um teste desenvolvido e validado nos Estados Unidos como parâmetro psicométrico de medida da criatividade de americanos e coreanos. Por outro lado, Wong e Niu (2013), avaliando a criatividade de americanos e chineses, aplicaram metodologias múltiplas, sendo que em uma delas os trabalhos criativos foram avaliados por juízes especialistas de diferentes nacionalidades, buscando atender três exigências descritas por Amabile (1996). Em primeiro lugar, os testes não dependiam de habilidades especializadas que alguns participantes poderiam ter cultivado mais do que outros (por exemplo, fotografia, escultura). Em segundo, os testes eram abertos para permitir flexibilidade e novidade. $\mathrm{E}$, em terceiro, foram solicitados produtos identificáveis para que os juízes avaliassem sua criatividade. Ainda que por meio de aparato multimetodológico e com a avaliação de juízes multinacionais, os americanos tiveram melhor desempenho criativo. 0 estudo revelou também que chineses tiveram melhor desempenho em tarefas que exigiam habilidades de pensamento dedutivo. Os autores relacionaram os resultados de desempenho nas tarefas aos estereótipos, identificados na pesquisa, segundo os quais os americanos são mais criativos e chineses mais dedutivos (Wong \& Niu, 2013).

Muito embora a maioria dos estudos de comparação tenham indicado uma superioridade criativa ocidental, $\mathrm{Hu}$, Shen, Lin e Adey (2010) verificaram que orientais podem ter desempenho superior em uma das facetas da criatividade. Investigando a criatividade científica em uma amostra de 1.190 adolescentes britânicos no Reino Unido e 1.087 adolescentes chineses na China, descobriram que os adolescentes chineses superaram os britânicos na resolução de problemas criativos, ao passo que os adolescentes britânicos superaram os chineses em quase todos os outros aspectos da criatividade científıca, como imaginação criativa, experimento científico e design de produto.

Para além das pesquisas citadas, um indicador global destaca-se na comparação entre feitos inovadores do Ocidente e do Oriente. A patente, entendida como propriedade, é a garantia de um direito exclusivo por uma invenção, na qual um produto ou um processo promove uma nova forma de fazer algo ou oferece uma nova solução técnica para um problema (Organização Mundial de Propriedade Intelectual OMPI, 2017). Amabile (1988) argumenta que a inovação deriva da implementação bem-sucedida de ideias criativas, e que esse termo é especialmente utilizado em organizações. Nesse sentido, associa-se o registro de patentes de produtos inovadores como propriedade de um feito criativo.

Países asiáticos têm despontado no ranking de patentes divulgado anualmente pela OMPI. No último relatório divulgado pela OMPI (2016), a China lidera o quadro de patentes registradas em 2015, com mais de um milhão e cem registros. 


\section{INTERACÃO EM LF PSICOLOGIA}

Os Estados Unidos aparecem em segundo lugar com quase 600 mil registros. Sendo países populosos, poder-se-ia argumentar que o alto número de patentes registradas pelo primeiro e segundo lugares é decorrente de suas populações expressivas. No entanto, em terceira e quarta posição voltam a surgir países asiáticos, Japão e Coreia, com 300 e 200 mil patentes registradas, o que coloca os países do Oriente em lugar de maior representatividade no registro de ideias inovadoras em comparação com os do Ocidente.

Assim, embora pesquisas sobre o potencial criativo em diferentes países tenham indicado que ocidentais parecem ser mais criativos que orientais, o fato de que algumas valeramse de uma definição e mensuração da criatividade apenas na perspectiva americana psicométrica pode ser um viés. Além disso, há pesquisas que demonstram a falibilidade da referida superioridade e, a partir da perspectiva de registro de produtos inovadores, os orientais levam vantagem sobre os ocidentais.

A análise da definição do conceito e a decorrente comparação entre os dois polos está longe de se esgotar. Destacase, no entanto, que a análise do desempenho criativo de um indivíduo deve, primeiramente, considerar o que é criatividade naquele contexto. $\mathrm{O}$ ato criativo não existe isoladamente, ele é inerentemente interativo e dependente do contexto (Csikszentmihalyi, 1996). A cultura influencia as pessoas criativas, o ambiente e o entendimento sobre o que é criatividade naquele contexto, de forma recíproca e dinâmica.

\section{CONSIDERAÇÕES FINAIS}

Dados históricos e concepções atuais, implícitas e explícitas, demonstram o quanto o entendimento sobre a criatividade pode variar de acordo com a cultura. 0 presente trabalho abordou algumas perspectivas, ocidentais e orientais, da criatividade humana, sem delimitar, no entanto, um significado universal. Runco e Jaeger (2012) discutem uma definição padronizada de criatividade. Por meio de revisão da literatura, os autores observaram que dois critérios, embora empregados com distintas nomenclaturas, são recorrentes desde os anos 50 nas descrições teóricas da criatividade. Original e eficaz, também denominados como não usual, único, incomum, útil, ajustado, adequado; seriam os dois atributos presentes nessa proposta de estandardização do conceito de criatividade, baseada em descrições publicadas ao longo dos anos. Para além de características ou de uma definição universal, deve-se considerar que a criatividade se manifesta de diferentes modos em distintas culturas (Simonton \& Ting, 2010).

Csikszentmihalyi (1999) apresenta uma abordagem contextual e um contraponto à discussão sobre a definição desse termo, questionando o fato dos pesquisadores buscarem ordinariamente responder o que é a criatividade. De acordo com o autor, ao invés de definir a criatividade, o importante seria descobrir onde ela está, ou, ainda, mais especificamente, verificar como o produto criativo é incorporado à cultura. Para isso, não se deve analisar isoladamente as pessoas ou os produtos, porque a criatividade não é o resultado da ação de um indivíduo por si só, mas é produto da interação de três forças: a pessoa, o domínio e o campo.

A pessoa é quem cria a ideia ou o produto. Ela é influenciada por sua bagagem genética e suas experiências pessoais. Destarte, destacam-se três aspectos pessoais que podem favorecer a criatividade de uma pessoa: as características cognitivas e afetivas, o conhecimento sobre a área e o contexto. 0 modo como a pessoa relaciona-se com o ambiente influencia o produto criativo. 0 domínio é composto por regras e procedimentos simbólicos compartilhados pela sociedade. Tratase de uma área de especialidade preexistente, que pode ser abrangente, como arte e matemática, ou específica, como teoria dos jogos e semiótica. 0 domínio é estabelecido culturalmente e perdura, preservando ou transmitindo ideias para as próximas gerações. Domínios que têm informações claras, acessíveis e conectadas e que ocupam posição central na cultura propiciam inovações e produtos criativos, diferentemente de domínios pouco estruturados. Um produto criativo gera mudança no domínio. Para alterar um domínio, entretanto, um produto precisa ser avaliado e aceito por especialistas, como professores, críticos, formadores de opinião, que compõem o conceito de campo. Um campo rígido, defensivo ou imerso em um sistema social pode desencorajar a criatividade. Os critérios de avaliação do campo podem variar ao longo do tempo (Csikszentmihalyi, 1996, 1999).

Em sua teoria sistêmica, Csikszentmihalyi (1996) caracteriza o ato criativo como inerentemente interativo e dependente do contexto. Assim, a cultura influencia as pessoas criativas, os membros do domínio e os guardiões do campo, de forma recíproca e dinâmica. Embora a negligência das influências culturais no estudo da criatividade tenha sido reconhecida no meio acadêmico, em alguns casos, pesquisas recentes ainda subestimam o papel do contexto e da cultura no desenvolvimento, na expressão e na mensuração da criatividade pelo mundo (Kaufman \& Sternberg, 2006; Lubart, 2010). Diante desse reconhecimento, espera-se com este estudo contribuir para que pesquisas em criatividade levem em conta a cultura e o contexto, especialmente em estudos que comparam o desempenho criativo de pessoas.

Sem um ambiente culturalmente determinado, não se pode sequer iniciar um processo criativo. As ideias e as informações que uma pessoa criativa explora existem no sistema simbólico de uma cultura antes que se imagine um produto 


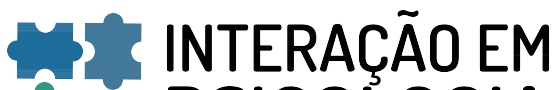 ET PSICOLOGIA}

criativo. Tanto uma ideia genuinamente inovadora (criatividade-H), que nunca tenha sido pensada na história humana, como uma ideia valiosa apenas no âmbito do indivíduo que a propõe (criatividade- $P$ ) derivam de referências tácitas ou explícitas a algum sistema gerativo específico e de suas restrições (Boden, 1994). Assim, segundo Csikszentmihalyi (1988), a única forma de estudar a criatividade é por meio de comparação, avaliação e interpretação dentro e entre culturas. A proposta teórica desse autor dispõe de instrumentos conceituais que possibilitam sistematizar e avaliar a influência dos fatores sociais, culturais e históricos na produção e na avaliação da criatividade, em uma perspectiva que ultrapassa os limites do indivíduo singularizado. Nesse sentido, dá-se um passo adiante da mera constatação da importância e influência da cultura no processo criativo para a delimitação de escopo e metodologia que consideram o fenômeno criativo em meio a todo um sistema cultural e social complexo.

De outro lado, Simonton e Ting (2010) argumentam que a perspectiva historiométrica, mais que qualquer outra metodologia científica, fornece ferramentas para avaliar a criatividade individual e coletiva considerando a história e a cultura de uma civilização ou nação. Os autores realizaram uma revisão de achados historiométricos ocidentais e orientais, comparando variáveis coletivas e individuais utilizadas para analisar a criatividade de alto impacto. Entre os critérios coletivos, foram comparados: disponibilidade de modelos, guerras, instabilidade política, fragmentação política, distúrbios civis e ideologia. Os critérios individuais foram: precocidade, produtividade, idade, versatilidade, longevidade, educação formal, doença mental. Os resultados evidenciaram que a criatividade individual difere pouco de uma civilização para outra, mas as circunstâncias socioculturais induzem divergências a nível coletivo.

Por meio dos critérios propostos pela historiometria, é possível medir a criatividade como fenômeno social, influenciado por aspectos diversos: ambientais, políticos, ideológicos, biológicos, personológicos, entre outros. Assim, dado que as civilizações ocidentais e orientais possuem um alto grau de consciência histórica e que as variadas circunstâncias socioculturais influenciam a atividade criativa, a perspectiva historiométrica é uma alternativa possível para o estudo intercultural da criatividade.

\section{CONTRIBUIÇÃO DE CADA AUTOR}

M. P. R. e D. S. F. contribuíram para a conceitualização, investigação e visualização do artigo; M. P. R. foi responsável pela redação inicial do artigo (rascunho) e D. S. F foi responsável pela redação final (revisão e edição)
Marina Porto-Ribeiro e Denise de Souza Fleith

\section{DECLARAÇÃO DE CONFLITOS DE INTERESSES}

As autoras declaram que não há conflitos de interesse no manuscrito submetido

\section{REFERÊNCIAS}

Albert, R. S., \& Runco, M. A. (1999). A history of research on creativity. In R. J. Sternberg (Ed.), Handbook of Creativity, (pp. 16-31). New York, NY: Cambridge University Press.

Alencar, E. M. L. S., \& Fleith, D. S. (2009). Criatividade. Múltiplas Perspectivas. Brasília, DF: Editora UnB

Amabile, T.M. (1983). The Social Psychology of Creativity. New York, NY: Springer.

Amabile, T. M. (1988). A model of creativity and innovation in organizations. In B. M. Staw \& L. L. Cummings (Eds.), Research in Organizational Behavior (Vol. 10, pp. 123-167). Greenwich, CT: JAI Press.

Amabile, T. (1996). Creativity in Context. Boulder, CO: Westview Press.

Barron, F. (1955). The disposition toward originality. The Journal of Abnormal and Social Psychology, 51, 478-485.

Bellanca, J., \& Brandt, R. (2010). 21st Century Skills: Rethinking How Students Learn. Bloomington, IN: Solution Tree Press.

Boden, M. (1994). Dimensions of Creativity. Cambridge, MA: MIT Press.

Chang, C. Y. (1970). Creativity and Taoism: A Study of Chinese Philosophy, Art, \& Poetry ( $1^{\text {a }}$ ed.). New York, NY: Harper \& Row.

Cheng, C. Y. (1991). New Dimensions of Confucian and NeoConfucian Philosophy. Albany, NY: State University of New York Press.

Cropley, D. H. (2010). Malevolent innovation: Opposing the dark side of creativity. In D. H. Cropley, A. J. Cropley, J. C. Kaufman, \& M. A. Runco (Eds.), The Dark Side of Creativity, (pp. 339-359). New York, NY: Cambridge University Press.

Csikszentmihalyi, M. (1988). Society, culture, and person: A systems view of creativity. In R. J. Sternberg (Ed.), The Nature of Creativity (pp. 325-339). New York, NY: Cambridge University Press.

Csikszentmihalyi, M. (1996). Creativity. New York, NY: HarperCollins.

Csikszentmihalyi, M. (1999). Implications of a systems perspective for the study of creativity. In R. J. Sternberg (Ed.), Handbook of Creativity (pp. 313-335). New York, NY: Cambridge University Press.

Csikszentmihalyi, M., \& Wolfe, R. (2000). New conceptions and research approaches to creativity: Implications of a systems perspective for creativity in education. In K. A. 


\section{H NTERAC̄öEM ET PSICOLOGIA}

Heller, F. J. Monks, R. J. Sternberg, \& R. F. Subotnik (Eds.), International Handbook of Giftedness and Talent (pp. 8193). Oxford, UK: Elsevier Science.

Eisenman, R. (2010). Creativity and crime: How criminals use creativity to succeed. In D. H. Cropley, A. J. Cropley, J. C. Kaufman, \& M. A. Runco (Eds.), The Dark Side of Creativity (pp. 204-217). New York, NY: Cambridge University Press.

Gino, F., \& Arieli, D. (2012). The dark side of creativity: Original thinkers can be more dishonest. Journal of Personality and Social Psychology, 102, 445-459. http://dx.doi.org/10.1037/ a0026406

Glaveanu, V. P. (2010). Paradigms in the study of creativity: Introducing the perspective of cultural psychology. New Ideas in Psychology, 28, 79-93. http://dx.doi.org/10.1016/ j.newideapsych.2009.07.007

Guilford, J. P. (1967). The Nature of Human Intelligence. New York, NY: McGraw-Hill.

Hennessey, B. (2003). The social psychology of creativity. Scandinavian Journal of Educational Research, 47, 253271. http://dx.doi.org/10.1080/00313830308601

Hondzel, C. D., \& Gulliksen, M. S. (2015). Culture and creativity: Examining variations in divergent thinking within norwegian and canadian communities. Sage Open, 5, 1-13. http://dx.doi.org/10.1177/2158244015611448

Hu, W., Shen, J., Lin, C., \& Adey, P. (2010, July). The comparisons of the development of scientific creativity between english and chinese adolescents. In W. Niu \& J. C. Kaufman (Co-Chair), Creativity of Chinese and their Western counterparts. Simpósium conducted at 118a American Psychological Association 118th Annual Convention, San Diego, CA. Retrieved from http://e.ctpad.net/upload/files/ HWP/hy-021.pdf

Kaufman, A. B., Butt, A. E., Kaufman, J. C., \& Colbert-White, E. N. (2011). Towards a neurobiology of creativity in nonhuman animals. Journal of Comparative Psychology, 125, 255-272. http://dx.doi.org/10.1037/a0023147

Kaufman, J. C., \& Sternberg, R. J. (Eds). (2006). The International Handbook of Creativity. New York, NY: Cambridge University Press.

Kim, K. H., Lee, H. E., Chae, K., Anderson, L., \& Laurence, C. (2011). Creativity and Confucianism among American and Korean educators. Creativity Research Journal, 23, 357371.http://dx.doi.org/10.1080/10400419.2011.621853

Klausen, S. H. (2010). The notion of creativity revisited: A philosophical perspective on creativity research. Creativity Research Journal, 22, 347-360. http://dx.doi.org/ $10.1080 / 10400419.2010 .523390$

Krausz, L. S. (2007). As Musas: Poesia e Divindade na Grécia Arcaica. São Paulo, SP: Edusp.

Lau, S., Hui, A., \& Ng, G. (2004). Creativity: When East Meets West. Singapore: World Scientific.
Lan, L., \& Kaufman, J. C. (2012). American and Chinese similarities and differences in defning and valuing creative products. Journal of Creative Behavior, 46, 285-306. http:// dx.doi.org/10.1002/jocb.19

Lubart, T. (1999). Creativity across cultures. In R. J. Sternberg (Ed.), Handbook of Creativity (pp. 339-350). New York, NY: Cambridge University Press.

Lubart, T. (2010). Cross-cultural perspectives on creativity. In C. Kaufman \& R. J. Sternberg (Eds.), The Cambridge Handbook of Creativity (pp. 265-278). New York, NY: Cambridge University Press.

MacKinnon, D. W. (1962). The nature and the nurture of creative talent. American Psychologist, 17, 484-495.

Nakamura, J., \& Csikszentmihalyi, M. (2014). The concept of flow. In M. Csikszentmihalyi (Ed.), Flow and the Foundations of Positive Psychology (pp. 239-263). Chicago, IL: Springer Netherlands.

Niu, W., \& Sternberg, R. (2002). Contemporary studies on the concept of creativity: The east and the west. Journal of Creative Behavior, 36, 269-288. http://dx.doi.org/ 10.1002/j. 2162-6057.2002.tb01069.x

Niu W., \& Sternberg R. J. (2006). The philosophical roots of western and eastern conceptions of creativity. Journal of Theoretical and Philosophical Psychology, 26, 18-38. http:// dx.doi.org/10.1037/h0091265

Organização Mundial de Propriedade Intelectual - OMPI (2016, Novembro). Innovation Flying High. Retrieved from: http://www.wipo.int/ipstats/en/infographics/wipi_2016/

Organização Mundial de Propriedade Intelectual - OMPI(2017, Maio). Frequently asked questions: Patents. Retrieved from: http://www.wipo.int/patents/en/ faq_patents.html

Porto-Ribeiro, M. M., \& Fleith, D. S. (no prelo). Criatividade e multiculturalismo: Revisão de literatura. Temas em Psicologia, 26.

Rudowicz, E., \& Hui, A. (1997). The creative personality: Hong Kong perspective. Journal of Social Behavior \& Personality, $12,139-157$

Rudowicz, E., \& Yue, X. (2000). Concepts of creativity: Similarities and differences among mainland, Hong Kong and Taiwanese Chinese. Journal of Creative Behavior, 34, 175192. 6057.2000.tb01210.x

Runco, M. S. (2017). The dark side of creativity. Potential better left unfulfilled. In J. A. Plucker (Ed.), Creativity and Innovation. Theory, Research, and Practice (pp. 49-59). Waco, TX: Prufrock Press.

Runco, M. A., \& Jaeger, G. J. (2012). The standard definition of creativity. Creativity. Research Journal, 24, 92-96. https:// doi.org/10.1080/10400419.2012.650092 


\section{-4: INTERACÃO EM IT PSICOLOGIA}

Runco, M. A., Millar, G., Acar, S., \& Cramond, B. (2010). Torrance Tests of Creative Thinking as predictors of personal and public achievement: A fifty year follow up. Creativity Research Journal, 22, 361-368. http://dx.doi.org/ $10.1080 / 10400419.2010 .523393$

Simonton, D. K. (1994). Greatness. Who Makes History and Why. New York, NY: The Guilford Press.

Simonton, D. K., \& Ting, S. S. (2010). Creativity in eastern and western civilizations: The lessons of historiometry. Management and Organization Review, 6, 329-350. http://dx.doi.org/ 10.1111/j.1740-8784.2010.00188.x

Sternberg, R. J. (1985). Implicit theories of intelligence, creativity, and wisdom. Journal of Personality and Social Psychology, 49, 607-627. http://dx.doi.org/ 10.1037/0022-3514.49.3.607

Sternberg, R. J., \& Lubart, T. I. (1995). Defying the Crowd: Cultivating Creativity in a Culture of Conformity. New York, NY: Free Press.

Sternberg, R. J., \& Lubart, T. I. (1999). The concept of creativity: Prospects and paradigms. In R. J. Sternberg (Ed.), Handbook of Creativity (pp. 3-15). New York, NY: Cambridge University Press.

Sternberg, R. J., Jarvin, L., \& Grigorenko, E. (2009). Teaching for Wisdom, Intelligence, Creativity and Success. Thousand Oaks, CA: Sage.

Torrance, E. (1988). The nature of creativity as manifest in its testing. In R. Sternberg (Ed.), The Nature of Creativity: Contemporary Psychological Perspectives (pp. 43-75). New York, NY: Cambridge University Press.
Marina Porto-Ribeiro e Denise de Souza Fleith

Trilling, B., \& Fadel, C. (2009). Twenty-first Century Skills: Learning for Life in our Times. San Francisco, CA: Jossey-Bass.

Tsang, A. S. L., \& Prendergast, G. (2009). Does culture affect evaluation expressions? A cross-cultural analysis of chinese and american computer game reviews. European Journal of Marketing, 43, 686-707. http://dx.doi.org/ 10.1108/03090560910947007

Weiner, R. (2000). Creativity and Beyond: Cultures, Values, and Change. Albany, NY: State University of New York Press.

Wong, R., \& Niu, W. (2013). Cultural difference in stereotype perceptions and performances in non-verbal deductive reasoning and creativity. Journal of Creative Behavior, 47, 4159. http://dx.doi.org/10.1002/jocb.22

Zha, P., Walczyk, J. J., Griffith-Ross, D. A., Tobacyk, J. J., \& Walczyk, D. F. (2006). The impact of culture and individualism-collectivism on the creative potential and achievement of american and chinese adults. Creativity Research Journal, 18, 355-366. http://dx.doi.org/10.1207/ s15326934crj1803_10

Submetido em 29/09/2017 Primeira decisão editorial em 04/12/2017 Aceito em 12/12/2017 